\title{
Myocardial regeneration strategy using Wharton's jelly mesenchymal stem cells as an off-the-shelf 'unlimited' therapeutic agent: results from the Acute Myocardial Infarction First-in-Man Study
}

\author{
Piotr Musialek ${ }^{1,2}$, Adam Mazurek ${ }^{1,2}$, Danuta Jarocha ${ }^{3}$, Lukasz Tekieli2,4, Wojciech Szot ${ }^{5}$, \\ Magdalena Kostkiewicz ${ }^{2,5}$, R. Pawel Banys ${ }^{6}$, Malgorzata Urbanczyk ${ }^{6}$, Andrzej Kadzielski ${ }^{4}$, Mariusz Trystula², \\ Jacek Kijowski ${ }^{3}$, Krzysztof Zmudka ${ }^{2,4}$, Piotr Podolec ${ }^{1,2}$, Marcin Majka ${ }^{3}$ \\ ${ }^{1}$ Department of Cardiac and Vascular Diseases, Jagiellonian University Medical College, Krakow, Poland \\ ${ }^{2}$ Clinical Departments, John Paul II Hospital, Krakow, Poland \\ ${ }^{3}$ Department of Transplantation, Jagiellonian University Medical College, Krakow, Poland \\ ${ }^{4}$ Department of Interventional Cardiology, Jagiellonian University Medical College, Krakow, Poland \\ ${ }^{5}$ Nuclear Imaging Laboratory, John Paul II Hospital, Krakow, Poland \\ ${ }^{6}$ Magnetic Resonance Imaging Laboratory, John Paul II Hospital, Krakow, Poland
}

Postep Kardiol Inter 2015; 11, 2 (40): 100-107

DOI: $10.5114 /$ pwki.2015.52282

\section{A bstract}

Introduction: In large-animal acute myocardial infarction (AMI) models, Wharton's jelly (umbilical cord matrix) mesenchymal stem cells (WJMSCs) effectively promote angiogenesis and drive functional myocardial regeneration. Human data are lacking.

Aim: To evaluate the feasibility and safety of a novel myocardial regeneration strategy using human WJMSCs as a unique, allogenic but immuno-privileged, off-the-shelf cellular therapeutic agent.

Material and methods: The inclusion criterion was first, large (LVEF $\leq 45 \%, \mathrm{CK}-\mathrm{MB}>100 \mathrm{U} / \mathrm{l}) \mathrm{AMI}$ with successful infarct-related artery primary percutaneous coronary intervention reperfusion ( $\mathrm{TIMI} \geq 2$ ). Ten consecutive patients (age 32-65 years, peak hs-troponin T $17.3 \pm 9.1 \mathrm{ng} / \mathrm{ml}$ and peak CK-MB $533 \pm 89 \mathrm{U} / \mathrm{l}$, sustained echo LVEF reduction to $37.6 \pm 2.6 \%$, CMRI LVEF $40.3 \pm 2.7 \%$ and infarct size $20.1 \pm 2.8 \%$ ) were enrolled.

Results: $30 \times 10^{6}$ WJMSCs were administered (LAD/Cx/RCA in 6/3/1) per protocol at $\approx 5-7$ days using a cell delivery-dedicated, coronary-non-occlusive method. No clinical symptoms or ECG signs of myocardial ischemia occurred. There was no epicardial flow or myocardial perfusion impairment (TIMI-3 in all; CTFC $45 \pm 8$ vs. $44 \pm 9, p=0.51$ ), and no patient showed hs-troponin T elevation $(0.92 \pm 0.29 \leq 24 \mathrm{~h}$ before vs. $0.89 \pm 0.28 \leq 24 \mathrm{~h}$ after; decrease, $p=0.04)$. One subject experienced, 2 days after cell transfer, a transient temperature rise $\left(38.9^{\circ} \mathrm{C}\right)$; this was reactive to paracetamol with no sequel. No other adverse events and no significant arrhythmias (ECG Holter) occurred. Up to 12 months there was one new, non-index territory lethal AMI but no adverse events that might be attributable to WJMSC treatment.

Conclusions: This study demonstrated the feasibility and procedural safety of WJMSC use as off-the-shelf cellular therapy in human AMI and suggested further clinical safety of WJMSC cardiac transfer, providing a basis for randomized placebo-controlled endpoint-powered evaluation.

Key words: myocardial regeneration, Wharton's jelly mesenchymal stem cells, human umbilical cord matrix, acute myocardial infarction, first-in-man, safety, feasibility.

\section{Introduction}

Current cellular treatment strategies aimed to stimulate cardiac regeneration in human acute myocardial infarction (AMI) suffer from lack of highly potent therapeutic cells available ad hoc in sufficient numbers [1].
Wharton's jelly mesenchymal stem cells (WJMSCs) are a small population of multipotent progenitor cells naturally present in the human umbilical cord matrix [2]. In the ongoing search for the best stem cell to stimulate myocardial regeneration in AMI [3], WJMSCs are an

Corresponding authors:

Piotr Musialek MD, DPhil FESC, Department of Cardiac and Vascular Diseases, Medical College Jagiellonian University, John Paul II Hospital, 80 Pradnicka St, 31-202 Krakow, Poland, e-mail: pmusialek@szpitaljp2.krakow.pl

Marcin Majka PhD DSc, Department of Transplantation, Medical College Jagiellonian University, Polish-American Institute of Pediatrics, 265 Wielicka St, 30-663 Krakow, Poland, e-mail: mmajka@cm-uj.krakow.pl

Received: 5.03.2015, accepted: 8.06.2015. 
attractive candidate [4] for a readily available "off-theshelf" cellular regenerative product for human use for several reasons. First, the umbilical cord is one of the most easily reached stem cell sources in the absence of ethical concerns associated with using embryo-derived stem cells $[5,6]$. Secondly, WJMSCs spontaneously secrete numerous pro-angiogenic factors, such as vascular endothelial growth factor (VEGF), angiopoietin-1, HGF and transforming growth factor $\beta 1$ (TGF- $\beta 1$ ) [7], and the release of pro-angiogenic molecules is accompanied by evidence of functional angiogenic potency in the infarcted mammalian heart [7-10]. Furthermore, WJMSCs show spontaneous expression of a number of early cardiac transcription factors in addition to their natural expression of core markers of undifferentiated human embryonic stem cells [11]. Wharton's jelly mesenchymal stem cells are more efficient in terms of stem cell potency than, for instance, bone marrow-derived stem cells predominantly used to date [5].

Wharton's jelly mesenchymal stem cells are naturally chemoattracted to the cardiac tissue, and they possess functional ability to populate the ventricular myocardium [12]. Indeed, in animal models, WJMSCS effectively induce angiogenesis [8-10] and show spontaneous differentiation into cardiac myocytes and endothelial cells $[8,9,13]$, a process consistent with the early findings in cardiomyocyte-conditioned medium WJMSC cultures [2]. In addition, recent evidence shows that WJMSCS can be successfully expanded ex vivo with high genomic stability, full clonogenic potential and full multilineage differentiation capacity, and with fully functional angiogenic potency, yielding large quantities of therapeutic cells available "when needed" and in "as-needed" numbers [7].

Wharton's jelly mesenchymal stem cells use is free of the logistic problems of autologous cell harvest in clinically unstable patients and circumvents the problem of the generally low (and variable) autologous regenerative cell yields $[14,15]$. This may be particularly relevant in $\mathrm{AMI}$, when the biologic window for therapeutic cell delivery does not allow any long-term culture and/or cell manipulation or lineage-specific expansion of autologous cells [16]. Although WJMSCs are a natural candidate for application in the setting of AMI in man, human feasibility and safety data are lacking $[17,18]$ (NB. PubMed exploration using various combinations of the terms "Wharton's jelly, umbilical cord matrix, infarction, heart, cardiac, myocardial, clinical, experimental, man or human"; last search performed on 20 May 2015).

\section{Aim}

The aim of the present work was to evaluate the feasibility and safety of a novel myocardial regeneration strategy using WJMSCs as an off-the-shelf 'unlimited' therapeutic agent.

\section{Material and methods}

Patient enrollment, infarct size data and cardiac imaging

Consecutive patients with successful infarct-related artery (IRA) stent-assisted primary percutaneous coronary intervention $(\mathrm{pPCl})$ reperfusion $(\mathrm{TIMI} \geq 2$ ) in large AMI, defined as sustained left ventricular ejection fraction (LVEF) reduction to $\leq 45 \%$ and peak creatine $\mathrm{ki}$ nase-MB (CK-MB) level exceeding $100 \mathrm{U} / \mathrm{l}$ (upper limit of normal $(U L N)<24 \mathrm{U} / \mathrm{l})$, were screened for enrollment as described in our earlier protocols $[14,15]$ except that the present study group was not limited to left anterior descending coronary artery (LAD) infarcts. Also, in the present study patients with two-vessel disease could be enrolled, but full coronary revascularization was required prior to cell transfer. No patients with three-vessel disease were recruited. The study was compliant with the Declaration of Helsinki and it was approved by the local Ethics Committee. All subjects provided informed written consent.

Echocardiography, gadolinium-enhanced cardiac magnetic resonance imaging (CMRI) and ECG-gated single photon emission computed tomography (SPECT) were performed within 24-48 $\mathrm{h}$ prior to cell transfer as described in our prior work [15].

\section{Human WJMSCs harvest and expansion}

The cells were harvested and prepared as described previously $[2,5,7,10]$. Umbilical donations were obtained following informed written consent, consistent with Directive 2004/23/EC, which sets the standards of quality and safety for the donation, procurement, testing, processing, preservation, storage, and distribution of human tissues and cells. Cell preparation was consistent with the International Conference on Harmonization and the US Food and Drug Administration (FDA) guidelines on stem cell-based therapies [19]. All solutions were prepared under Good Manufacturing Practice and all procedures were performed in line with Good Laboratory Practice.

In brief, MSCs were isolated using an enzymatic digestion strategy. Isolated cells were plated into $75 \mathrm{~cm}^{2}$ tissue culture flasks (Sarstedt Stare Babice, Poland) in Dulbecco's modified Eagle's medium (PAA Laboratories $\mathrm{GmbH}$, Goetzis, Austria) supplemented with 10\% FBS (PAA Laboratories GmbH, Goetzis, Austria), PDGF (PeproTech Inc, Rocky Hill, USA) and EGF (PeproTech Inc, Rocky Hill, USA) growth factors. Flasks were incubated at $37^{\circ} \mathrm{C}$ in a humidified atmosphere containing $5 \% \mathrm{CO}_{2}$, and after 7 days the medium was changed. At confluence, the adherent cells were detached using $0.25 \%$ trypsin and re-seeded at $0.075 \times 10^{6}$ cells $/ 75 \mathrm{~cm}^{2}$ flask and incubated again until confluence with a weekly medium change. After the second passage, cells were collected and frozen in $2.5 \times 10^{6}$ aliquots in liquid nitrogen. Five-seven days 
before treatment cells were thawed and expanded to obtain at least $30 \times 10^{6}$ cells.

\section{Wharton's jelly mesenchymal stem cells} preparation for human application

\section{Phenotypic evaluation of cultured WJMSCs}

The MSC phenotype was analyzed with antibodies specific for MSCS (CD73, CD90, and CD105) and hematopoietic cells (CD45) (all antibodies from BD Biosciences, San Jose, USA) using a FACSCalibur cytometer with CellQuest software (BD Biosciences Immunocytometry Systems, San Jose, USA). Wharton's jelly mesenchymal stem cells used for transplantation were $\geq 95 \%$ positive for CD73, CD90, CD105 and negative for CD45, and their proliferative and functional capacity was evaluated.

\section{Evaluation of cytogenetic stability of cultured WJMSCs}

Cytogenetic stability of cultured WJMSCs was confirmed via GTG banding. Metaphases were analyzed under an Olympus BX51 microscope with a camera (Olympus Corporation, Tokyo, Japan) to document photomicrographs. The CytoVision program (Leica Microsystems Inc., Buffalo Grove, USA) was used to arrange chromosomes into a karyogram.

\section{Sterility evaluation and obtaining single-cell suspension}

Sterility of the material was confirmed via microbiological evaluation. Immediately prior to transfer to the cathlab, WJMSCs were filtered to eliminate any potential cellular conglomerates that might cause microvascular plugging [20] and were suspended in $2 \mathrm{ml}$ of $0.9 \% \mathrm{NaCl}$ (B. Braun, Melsungen, Germany). In addition, the cells were gently tottered throughout the transport period to minimize any formation of cellular aggregates.

\section{Trans-IRA cell administration}

Cell administration to the infarct zone was performed using the previously described non-coronary occlusive technique for transcoronary delivery of cellular therapies $[14,21,22] .30 \times 10^{6}$ WJMSCS were re-suspended in $30 \mathrm{ml}$ of $0.9 \%$ saline and were administered via the IRA using a cell-delivery perfusion catheter [14, 15], using a filter to eliminate any potential cellular conglomerates. The cell suspension infusion was at $2 \mathrm{ml} / \mathrm{min}$, yielding $2 \times 10^{6}$ cells per minute.

\section{Clinical, angiographic and cardionecrotic marker evaluation}

Throughout the cell transfer, ECG was monitored carefully for any new ischemic changes or arrhythmias [14, 21]. Angiographic recordings were performed to evaluate the coronary epicardial flow and myocardial tissue perfusion using the established indices (TIMI grade, corrected TIMI frame count, cTFC) [23]. Hs-troponin T level (ULN $<0.014 \mathrm{ng} / \mathrm{ml}$ ) was determined $\leq 24 \mathrm{~h}$ before and $\leq 24 \mathrm{~h}$ after cell transfer.

\section{In-hospital follow-up and post-discharge patient monitoring}

Twenty-four hours ECG recordings were performed 24-48 h after cell administration. Close clinical vigilance has been applied for any adverse events up to 12 months (with further pre-specified points of analysis at every 12 months up to 5 years). A study-independent Safety Committee at the Jagiellonian University Department of Cardiac and Vascular Diseases performed initial event analysis in the context of the likelihood of the event association with cell transfer, and it oversaw any potential EC notification(s).

This work is consistent with the US Food and Drug Administration (FDA) guidelines on stem cell-based therapies [19].

\section{Statistical analysis}

Data are shown as mean \pm SEM (min.-max.) or number (\%). Two-tailed Student $t$-test was used for statistical comparisons (Statistica 10.0, StatSoft).

\section{Results}

Clinical characteristics of study patients and infarct size data are shown in Table I, indicating recruitment of a typical group of consecutive patients with a large, first acute myocardial infarction.

Except for the WJMSC transfer using a transradial approach at $\approx 5-7$ days after $\mathrm{pPCl}$, patient management was standard and was consistent with the European Society of Cardiology (ESC) guidelines. Myocardial imaging with SPECT and CMRI [15] was uncomplicated. No clinical complications occurred in the study group by the point of cell transfer.

No WJMSC genetic instability was detected and no or other laboratory-level safety issues occurred. Wharton's jelly mesenchymal stem cells transfer was performed per protocol in all study patients. No clinical symptoms or signs of myocardial ischaemia, and no new ischemic ECG changes occurred during or after WJMSC trans-IRA administration.

There was no indication of any negative effect of trans-IRA WJMSC transfer on epicardial or myocardial perfusion. In particular, the epicardial flow was TIMI-3 in all subjects before and after cell transfer, and CTFC was $45 \pm 8$ (from 17 to 76 ) prior to cell transfer and 44 \pm 9 (from 15 to 77 ) after cell transfer $(p=0.51)$. Also, no patient showed a hs-troponin $\mathrm{T}$ rise after cell transcoronary WJMSC transfer. The mean hs-troponin T level was $0.92 \pm 0.29$ (from 0.04 to 2.64 ) within $24 \mathrm{~h}$ before vs. 0.89 
\pm 0.28 (from 0.03 to $2.52 \mathrm{ng} / \mathrm{ml}$ ) within $24 \mathrm{~h}$ after cell administration ( $p=0.04$ for hs-troponin $\mathrm{T}$ decrease).

One subject experienced, two days after cell transfer, a transient temperature rise to $38.9^{\circ} \mathrm{C}$; this was reactive to paracetamol, with no signs of infection and no clinical sequel. The association between this event and cell transfer was considered possible, although transient fever may occur in the AMI recovery phase without any apparent cause other than myocardial necrosis [24].

One other patient had vein inflammation at the site of venous line insertion that was managed in a typical manner, and another, treated empirically with amoxicillin due to chronic obstructive pulmonary disease infectious exacerbation, exhibited a typical picture of amoxicillin-associated skin rash that was responsive to steroid and antihistaminic administration and amoxicillin withdrawal. Any potential association between the two latter events and WJMSC administration was judged, at the level of the Safety Committee, to be highly unlikely.

All three events were reported to the local EC, and no reciprocal safety concern was raised. No other adverse events and no significant arrhythmias (ECG Holter) occurred.

At discharge, the patients were transferred to a routine post-AMI rehabilitation program. Up to 12 months there was one new, non-index territory AMI that led to death but no adverse events that might be attributable to WJMSC treatment.

In summary, the one and only adverse event that was considered potentially related to WJMSC administration was a transient temperature rise in 1 patient that was responsive to typical paracetamol treatment and resolved rapidly and without sequel.

\section{Discussion}

The principal finding from this work is the feasibility and procedural safety of WJMSC use as an off-the-shelf, transcoronary cellular allogenic therapy in AMI in man. Our study also suggests further, post-procedural clinical safety of WJMSC cardiac transfer in humans, opening a new avenue towards controlled outcome studies using WJMSCs in AMI and in other conditions associated with depletion of functional myocardial tissue.

\section{Regeneration therapies in the era of optimal pharmaco-mechanical AMI treatment: reversal of the damage as a logical step to follow maximized reduction of myocardial tissue loss}

Several lines of evidence suggest that, in AMI, conventional pharmacological and mechanical therapies may have already reached the ceiling of efficacy (or are very close to such) in their capacity to limit myocardial injury and reduce the infarct size [25]. The concept of myocardial injury reversal (as the next step to follow maximized limitation as explored and implemented over the last three decades) by stimulating myocardial regen-
Table I. Study group $(n=10)$ characteristics and myocardial infarction parameters

\begin{tabular}{|c|c|}
\hline Variable & Mean \pm SEM (min.-max.) or $n(\%)$ \\
\hline Age [years] & $55.6 \pm 3(32-65)$ \\
\hline Women & $5(50)$ \\
\hline Diabetes & $3(30)$ \\
\hline Prior MI & $0(0)$ \\
\hline $\operatorname{IRA}=L A D / C x / R C A$ & $6 / 3 / 1(60 / 30 / 10)$ \\
\hline Ilb/Illa rec. inhibitor (Abciximab) & $4(40)$ \\
\hline IRA DES/BVS & $9 / 1(90 / 10)$ \\
\hline IRA TIMI-3/TIMI-2* & $8 / 2(80 / 20)$ \\
\hline Peak hs-troponin T [ng/ml] & $17.3 \pm 9.1(5.2-102.1)$ \\
\hline Peak CK-MB [U/I] & $533 \pm 89(148-965)$ \\
\hline Echo LVEF [\%] & $37.6 \pm 2.6(21-43)$ \\
\hline MRI LVEF§ [\%] & $40.3 \pm 2.7(26-49)$ \\
\hline G-SPECT LVEF§ [\%] & $36.8 \pm 2.8(19-48)$ \\
\hline MRI infarct size" [\%] & $20.1 \pm 2.8(7-41)$ \\
\hline SPECT infarct size ${ }^{\#}[\%]$ & $33.7 \pm 3.1(18-52)$ \\
\hline
\end{tabular}

SEM - standard error of the mean, IRA - infarct-related artery, $L A D / C X / R C A$ left anterior descending/circumflex/right coronary artery, LVEF-left ventricular ejection fraction, echo - echocardiography, SPECT - single photon emission computed tomography, G-SPECT = gated SPECT. *At pPCI completion (NB. at the cell transfer point all IRAs showed TIMI-3 flow), ${ }^{\S} \leq 24 \mathrm{~h}$ from cell transfer to minimize the effect of myocardial stunning on LVEF as an acute index of infarct size [15], "expressed as proportion of left ventricular muscle volume (MRI) or as infarct extent (SPECT).

eration is gradually gaining evidence of feasibility in the experimental and clinical setting, and cellular therapies are a pillar of this concept [3]. Nevertheless - and disappointingly to those who have worked extensively to advance the field over the last years - the efficacy of cellular therapies today remains too modest for any systematic clinical application [1, 3, 26]. Apart from the room for further improvement of cell delivery strategies [14, 16], important limiting factors are (i) lack of identification of sufficiently potent and clinically safe cell types, and (ii) insufficient quantities of (in the majority of studies - autologous) regenerative cells and lack of their 'off-the-shelf' availability. Indeed, both experimental and clinical data indicate that the therapeutic effect of progenitor cells is dose (i.e. therapeutic cell number)-dependent [26-28]. Autologous non-expanded/engineered cell yields are usually rather small $[14,15]$, and cell harvesting in AMI is associated with important challenges of patient safety and logistics. On the other hand, it is likely that in AMI the optimal window for transcoronary-applied cellular therapies may be limited to the first $\approx 1-2$ weeks [14], restricting the logistics of any autologous cell expansion strategies [16]. For these reasons, an intensive search is currently ongoing for clinically safe strategies using high regenerative potential allogenic cells available readily, on 
an ad-hoc basis, and in sufficient numbers to address the clinical need to meaningfully reverse the myocardial injury burden that remains after successful AMI reperfusion in order to reduce the scar formation and adverse myocardial remodeling, preventing arrhythmic death and heart failure.

\section{Wharton's jelly mesenchymal stem cells} properties in relation to other regenerative cell types in cardiovascular medicine

Clinical translational research in the setting of $\mathrm{AMI}$ and heart failure to date has primarily employed autologous and non-expanded bone marrow cells [1, 26]. More recent approaches include lineage-engineered cells (applicable in a chronic but not in the acute setting) [16]) and mesenchymal cells [18]. The use of bone marrow hematopoietic stem cells or mesenchymal stem cells from the bone marrow, adult organs and fetuses for myocardial regeneration faces the disadvantages of invasive isolation, limited cell numbers and ethical constraints [6]. Allogenic cells, on the other hand, require no patient harvest and can be available in numbers larger than in the case of autologous cells. But allogenic cells elicit, in principle, an antigen-specific immune response of rejection by the recipient [29]. This is manifested by the host generation of specific anti-donor antibodies directed against the allogenic mesenchymal stem cells that may lead to immune-mediated elimination of the allogenic cells $[29,30]$. Nevertheless, allogenic mesenchymal cells have been used in the context of myocardial regeneration in animal models of AMI [18, 31] (where they were shown to home to the infarct border zone [31], similar to the bone marrow-derived hematopoietic $\mathrm{CD} 34^{+}$cells in humans [15, 32]), and there is at least one recent report of human cardiac use of allogenic bone marrow-derived mesenchymal stem cells [33]. The immune responses elicited by conventional allogenic mesenchymal cells are complex [29], and no long-term or large-scale safety data are available yet.

Apart from ethics, immuno-rejection is the principal biologic limiting factor with (naturally allogenic) embryonic stem cells [6], whereas induced pluripotent stem cells face the key hurdle of potential tumorigenesis [6]. In that context, WJMSCs are unique as they are non-controversial, can be harvested painlessly in abundance, are self-renewing and highly proliferative, possess stemness properties that last several passages in vitro, are multipotent but do not induce tumorigenesis even though they have a number of embryonic cell markers $[6,11]$ Expansion of WJMSCs is not associated with any loss of genetic stability [7], as these cells are not susceptible to spontaneous malignant transformation [34]. Importantly, WJMSCs do not express major histocompatibility complex class II (HLA-DR) antigens or co-stimulatory immune response-associated surface antigens CD40, CD80, and
CD86 [35]. When placed in an allogenic environment, WJMSCS are unable to elicit the response of immune-mediated rejection or proliferation of host immune cells [5, $30,35]$. Thus in contrast to other allogenic mesenchymal cell types [30], WJMSCs do not elicit an allogenic immune response of transplant rejection [5, 30, 35].

In short, WJMSCS are uniquely immune-privileged; they are not rejected by an allogenic host and thus their allogenic transplantation is associated with no need for immunosuppression [5, 30, 35]. In addition, WJMSCs can be safely expanded to "as needed" quantities [5, 7, 10], can be phenotypically selected on a large scale to create repositories of distinct WJMSC populations [36], and - if needed - can be further reprogrammed/engineered towards the desired cell lines [10, 13, 37]. Moreover, evidence is accumulating for WJMSCs' successful use in engineering biological cardiac tissues [38-40] and an allogenic non-rejectable bioartificial heart [41].

\section{Evidence for functional regenerative effect of WJMSCs in pre-clinical studies in AMI}

The unique combination of high angiogenic and cardiogenic potential of WJMSCs at bench $[7,11]$ with their low immunogenicity [35] makes these cells an important candidate for systematic in-vivo evaluation of myocardial regenerative capacity [4]. Indeed, recent evidence in a porcine model of $\mathrm{AMI}$ demonstrated functionally significant myocardial regeneration capacity of WJMSCs [9]. In particular, Zhang et al. [9] found that $40 \times 10^{6}$ WJMSCS transplanted by direct injections into the acutely infarcted area in the pig not only stimulated new vessel formation but also survived and differentiated into cardiomyocytes and endothelial cells and promoted recruitment and differentiation of native cardiac stem cells. In addition, WJMSC transplantation reduced apoptosis and fibrosis in the infarcted myocardium. A non-treated control group and a control group treated with placebo (phosphate-buffered saline) injections (sham procedure) were used as comparators. Overall, the multi-factorial regenerative effect of $40 \times 10^{6}$ WJMSCS on the infracted tissue in situ translated into enhancement of viable myocardium significant both statistically and functionally [9].

Taken together, evidence in large-animal AMI models shows, consistent with tissue data ex vivo $[2,11,12]$ and small-animal AMI data [8, 10, 13], a functionally significant effect of reduction of post-infarct adverse remodeling and improvement of left ventricular function.

\section{Choice of WJMSC dose for the human AMI study with transcoronary cell delivery}

Evidence in experimental models of AMI shows that the therapeutic effect of progenitor cells is, in principle, dose-dependent [27]. Clinical evidence is overall consistent with the animal work, as it indicates a positive correlation between the cell dose and the effect on LVEF 
measured by echocardiography [28] or magnetic resonance imaging [26], suggesting that maximized cell doses should be used in the clinical setting. Nevertheless, there is also evidence that transcoronary delivery of (too) large cell doses (such as in the order of $\approx 50-75$ million) can become harmful to myocardium via impairment of coronary blood flow in the IRA territory and producing microinfarcts that occur most likely via distal embolization and microvascular plugging [20, 28, 42, 43].

Not only the absolute cell dose but also the cell infusion rate may be associated with impairment of IRA flow and myocardial injury demonstrated by an increase in troponin levels and new microinfarcts on histopathology. In the sheep, an adverse effect of mesenchymal precursor cell trans-IRA infusion on epicardial flow associated with an increase in troponin levels and microinfarcts on histopathology occurred with doses exceeding 40 million cells and infusion rates $\geq 2.5$ million cells per minute, while lower cell doses and infusion rates had no effect on epicardial perfusion or troponin release/microinfarct formation [20]. While a maximal total cell dose of 37.5 million was found to cause no acute myocardial injury in the sheep model of acute myocardial infarction in one study [20], a pilot study by another group showed, in a similar model, no late gadolinium enhancement on cardiac magnetic resonance imaging and only a small rise in troponin with the mesenchymal cell dose of 25 million (which might be due to the use of a coronary-occlusive rather than non-occlusive cell delivery system) but a substantial troponin rise and a substantial de novo late gadolinium enhancement occurred only with the dose of 75 million cells [42]. For these reasons, in the present human study, an additional $20 \%$ safety margin was applied against the apparent myocardial safety of the 37.5 million mesenchymal cell dose in the study by Houtgraaf et al. [20]. This resulted in our selection of the dose of 30 million WJMSCs and infusion rate of 2 million cells per minute for the human study protocol. Our results demonstrated that trans-IRA infusion of 30 million WJMSCs at the rate of 2 million cells per minute caused no deterioration of IRA epicardial flow (routine TIMI flow grade assessment) or tissue flow (cTFC evaluation). Moreover, no study subject showed a troponin increase $\leq 24 \mathrm{~h}$ after transcoronary cell transplantation.

Thus our findings are consistent with lack of any deleterious myocardial effect of WJMSC administration according to the present study protocol.

\section{Safety aspects of WJMSC therapy}

There are at least three aspects of the safety of WJMSC therapy: (1) biological safety of cell harvest, banking, ex-vivo expansion and preparation for human use; (2) procedural safety of WJMSC transcoronary transfer that needs to include minimized risk of myocardial necrosis through microvessel plugging; and (3) short-and long- term clinical safety of the allogenic biologic material use in humans.

The present study identified transient temperature rise in a temporal relation to WJMSC transcoronary transfer in one subject as the only adverse effect potentially associated with WJMSCS.

An association between WJMSC transfer and transient fever would be unsurprising, as this has been established in the mesenchymal stem cell clinical use literature [44]. Although no specific data in relation to WJMSCs seem to be available, this potential side effect has been reported using both autologous and allogenic mesenchymal cells, like in various human studies, and the overall odds ratio for transient fever was found to be at least 5-fold greater with mesenchymal cells than with placebo [44].

The temperature rise in a WJMSC-treated patient in our study was only transient, and it was fully responsive to paracetamol, leading to resolution without any sequel. As fever can occur in recent acute myocardial infarction in the absence of any cause other than myocardial necrosis (and the likelihood of its occurrence increases with the infarct size) [24], further work in a large patient cohort including WJMSC-exposed and non-exposed subjects is needed to establish the prevalence of transient body temperature rise in potential relation to transcoronary WJMSC transfer. Indeed, further clinical studies in larger patient samples will need to continue close monitoring for any potential clinically relevant side effects of cardiovascular regeneration therapies using WJMSCS.

\section{Limitations}

The modest number of study participants may be considered a relative limitation of our study. Nevertheless, the present work meets the criteria of a first-inman feasibility and safety study, while we believe that further patients should be evaluated already within the framework of a randomized controlled study with blinded analysis of potential therapeutic effects. Also, in the present study we did not perform any safety-focused WJMSC dose-response analysis. Apart from the requirement of large patient numbers for any dose-safety analysis, such potential evaluation was considered ethically unacceptable in the human setting. Rather, our choice of the WJMSC dose was based on careful analysis of the preclinical data (see above), and our selection involved the peak safe dose in large mammals with an additional $20 \%$ safety margin.

All in all, the lack of any negative effect of WJMSCS in the dose used in the present study on epicardial flow or myocardial tissue perfusion, and no troponin release in relation to cell transfer, are consistent with myocardial safety of the trans-IRA administration of 30 million WJMSCs as per the present human study protocol. 


\section{Conclusions and further directions}

This study demonstrated the feasibility and procedural safety of WJMSC use as off-the-shelf cellular therapy in human AMI, and it suggested WJMSC cardiac transfer clinical safety throughout the follow-up period.

The present findings justify further investigation of the therapeutic potential of WJMSCs in humans with large AMI within the framework of a randomized, placebo-controlled clinical study appropriately powered for pre-specified therapeutic endpoints. Consistent with the concept that regenerative cell effective uptake in the infarct area is an obligatory pre-requisite for seeking any therapeutic effect [15], further work needs to establish, as a bridge to the clinical outcome study, the magnitude of myocardial retention of WJMSCs in the therapeutic target zone in relation to that of other cell types used thus far in major clinical investigations [15, 17, 45].

\section{Acknowledgments}

K/ZDS/005644 (Jagiellonian University Medical College) and 265761 "CIRCULATE" (NCBR STRATEGMED, National Centre for Research and Development, Poland). Also supported by 'Gift of Hope' Regenerative Medicine Foundation in Krakow, 'For the Heart' Foundation in Krakow, and the John Paul II Hospital in Krakow, Poland.

\section{Conflict of interest}

The authors declare no conflict of interest.

\section{References}

1. Gyöngyösi M, Wojakowski W, Lemarchand P, et al. Meta-Analysis of Cell-based CaRdiac stUdiEs (ACCRUE) in patients with acute myocardial infarction based on individual patient data. Circ Res 2015; 116: 1346-60.

2. Wang HS, Hung SC, Peng ST, et al. Mesenchymal stem cells in the Wharton's jelly of the human umbilical cord. Stem Cells 2004; 22: 1330-7.

3. Badimon L, Vilahur G. Experimental cell therapy: the search for the best stem cell continues. J Am Coll Cardiol 2014; 64: 1695-7.

4. Corrao S, La Rocca G, Lo lacono M, et al. New frontiers in regenerative medicine in cardiology: the potential of Wharton's jelly mesenchymal stem cells. Curr Stem Cell Res Ther 2013; 8: 39-45.

5. Can A, Karahuseyinoglu S. Human umbilical cord stroma with regard to the source of fetus-derived stem cells. Stem Cells 2007; 25: 2886-95.

6. Bongso A, Fong CY. The therapeutic potential, challenges and future clinical directions of stem cells from the Wharton's jelly of the human umbilical cord. Stem Cell Rev 2013; 9: 226-40.

7. Swamynathan P, Venugopal P, Kannan S, et al. Are serum-free and xeno-free culture conditions ideal for large scale clinical grade expansion of Wharton's jelly derived mesenchymal stem cells? A comparative study. Stem Cell Res Ther 2014; 5: 88.

8. Wu KH, Zhou B, Yu CT, et al. Therapeutic potential of human umbilical cord derived stem cells in a rat myocardial infarction model. Ann Thorac Surg 2007; 83: 1491-8.
9. Zhang W, Liu XC, Yang L. Wharton's jelly-derived mesenchymal stem cells promote myocardial regeneration and cardiac repair after miniswine acute myocardial infarction. Coron Artery Dis 2013; 24: 549-58.

10. Santos Nascimento D, Mosqueira D, Sousa LM, et al. Human umbilical cord tissue-derived mesenchymal stromal cells attenuate remodeling after myocardial infarction by proangiogenic, antiapoptotic, and endogenous cell-activation mechanisms. Stem Cell Res Ther 2014; 5 : 5.

11. Gao LR, Zhang NK, Ding QA, et al. Common expression of stemness molecular markers and early cardiac transcription factors in human Wharton's jelly-derived mesenchymal stem cells and embryonic stem cells. Cell Transplant 2013; 22: 1883-900.

12. Lupu M, Khalil M, Andrei E, et al. Integration properties of Wharton's jelly-derived mesenchymal stem cells into ventricular slices of murine hearts. Cell Physiol Biochem 2011; 28: 63-76.

13. Yannarelli G, Dayan V, Pacienza N, et al. Human umbilical cord perivascular cells exhibit enhanced cardiomyocyte reprogramming and cardiac function after experimental acute myocardial infarction. Cell Transplant 2013; 22: 1651-66.

14. Musialek P, Tekieli L, Kostkiewicz M, et al. Randomized transcoronary delivery of $\mathrm{CD}_{3} 4^{+}$cells with perfusion versus stop-flow method in patients with recent myocardial infarction: early cardiac retention of ${ }^{99 m}$ Tc-labeled cells activity. J Nucl Cardiol 2011; 18: 104-16.

15. Musialek P, Tekieli L, Kostkiewicz M, et al. Infarct size determines myocardial uptake of $\mathrm{CD} 34^{+}$cells in the peri-infarct zone: results from a study of ${ }^{99 m} T c$-extametazime-labeled cell visualization integrated with cardiac magnetic resonance infarct imaging. Circ Cardiovasc Imaging 2013; 6: 320-8.

16. Bartunek J, Behfar A, Dolatabadi D, et al. Cardiopoietic stem cell therapy in heart failure: the C-CURE (Cardiopoietic stem Cell therapy in heart failURE) multicenter randomized trial with lineage-specified biologics. J Am Coll Cardiol 2013; 62: 2454-6.

17. Tongers J, Losordo DW, Landmesser U. Stem and progenitor cellbased therapy in ischaemic heart disease: promise, uncertainties, and challenges. Eur Heart J 2011; 32: 1197-206.

18. Karantalis V, Hare JM. Use of mesenchymal stem cells for therapy of cardiac disease. Circ Res 2015; 116: 1413-30.

19. Halme DG, Kessler DA. FDA regulation of stem-cell-based therapies. N Engl J Med 2006; 355: 1730-5.

20. Houtgraaf JH, de Jong R, Kazemi K, et al. Intracoronary infusion of allogeneic mesenchymal precursor cells directly after experimental acute myocardial infarction reduces infarct size, abrogates adverse remodeling, and improves cardiac function. Circ Res 2013; 113: 153-66.

21. Musialek P, Tracz W, Skotnicki AB, et al. Transcoronary stem cell delivery using physiological endothelium-targeting perfusion technique: the rationale and a pilot study involving a comparison with conventional over-the-wire balloon coronary occlusions in patients after recent myocardial infarction. Kardiol Pol 2006; 64: 489-98.

22. Musialek P, Kostkiewicz M, Banys RP, et al. Early myocardial engraftment of autologous $\mathrm{CD}_{3} 4^{+}$cells administered transcoronary via a physiological cell-delivery system. Eur J Nucl Med Mol Imaging 2008; 35: 1929-30.

23. Musialek P. Coronary thrombus management. In: Treatment strategies - interventional cardiology. Eeckhout E, Evangelista A, Holmes D, Leon MB, Stone GW (eds). Cambridge Research Centre, UK, 2012; 97-106.

24. Kacprzak M, Kidawa M, Zielinska M. Fever in myocardial infarction: is it still common, is it still predictive? Cardiol J 2012; 19 : 369-73. 
25. Musialek P. TASTE-less endpoint of 30-day mortality (and some other issues with TASTE) in evaluating the effectiveness of thrombus aspiration in STEMI: not the "evidence" to change the current practice of routine consideration of manual thrombus extraction. State-of-the-art review. Kardiol Pol 2014; 72: 479-87.

26. Clifford DM, Fisher SA, Brunskill SJ, et al. Stem cell treatment for acute myocardial infarction. Cochrane Database Syst Rev 2012; 2: CD006536.

27. Iwasaki H, Kawamoto A, Ishikawa M, et al. Dose-dependent contribution of CD34-positive cell transplantation to concurrent vasculogenesis and cardiomyogenesis for functional regenerative recovery after myocardial infarction. Circulation 2006; 113: 1311-25.

28. Gao LR, Pei XT, Ding QA, et al. A critical challenge: dosage-related efficacy and acute complication intracoronary injection of autologous bone marrow mesenchymal stem cells in acute myocardial infarction. Int J Cardiol 2013; 168: 3191-9.

29. Griffin MD, Ryan AE, Alagesan S, et al. Anti-donor immune responses elicited by allogeneic mesenchymal stem cells: what have we learned so far? Immunol Cell Biol 2013; 91: 40-51.

30. Lohan P, Coleman CM, Murphy JM, et al. Changes in immunological profile of allogeneic mesenchymal stem cells after differentiation: should we be concerned? Stem Cell Res Ther 2014; 5: 99.

31. Wojakowski W, Tendera M, Cybulski W, et al. Effects of intracoronary delivery of allogenic bone marrow-derived stem cells expressing heme oxygenase-1 on myocardial reperfusion injury. Thromb Haemost 2012; 108: 464-75.

32. Musialek P, Tracz W, Kostkiewicz M, et al. Visualisation of early engraftment of transcoronary applied $\mathrm{CD} 34^{+}$cells in the infarct border zone. Kardiol Pol 2008; 66: 73-7.

33. Hare JM, Fishman JE, Gerstenblith G, et al. Comparison of allogeneic vs autologous bone marrow-derived mesenchymal stem cells delivered by transendocardial injection in patients with ischemic cardiomyopathy: the POSEIDON randomized trial. JAMA 2012; 308: 2369-79.

34. Tang $\mathrm{Q}$, Chen $\mathrm{Q}$, Lai X, et al. Malignant transformation potentials of human umbilical cord mesenchymal stem cells both spontaneously and via 3-methycholanthrene induction. PLoS One 2013; 8: e81844.

35. Weiss ML, Anderson C, Medicetty S, et al. Immune properties of human umbilical cord Wharton's jelly-derived cells. Stem Cells 2008; 26: 2865-74.

36. Holley RJ, Tai G, Williamson AJ, et al. Comparative quantification of the surfaceome of human multipotent mesenchymal progenitor cells. Stem Cell Reports 2015; 4: 473-88.

37. Duya P, Bian Y, Chu X, et al. Stem cells for reprogramming: could hUMSCs be a better choice? Cytotechnology 2013; 65: 335-45.

38. Kadner A, Zund G, Maurus C, et al. Human umbilical cord cells for cardiovascular tissue engineering: a comparative study. Eur J Cardiothorac Surg 2004; 25: 635-41.

39. Breymann C, Schmidt D, Hoerstrup SP. Umbilical cord cells as a source of cardiovascular tissue engineering. Stem Cell Rev 2006; 2: 87-92.

40. Semenov OV, Breymann C. Mesenchymal stem cells derived from Wharton's jelly and their potential for cardio-vascular tissue engineering. Open Tissue Eng Regen Med J 2011; 4: 64-71.

41. Weymann A, Patil NP, Sabashnikov A, et al. Bioartificial heart: a human-sized porcine model - the way ahead. PLoS One 2014; 9: e111591.

42. Grieve SM, Bhindi R, Seow J, et al. Microvascular obstruction by intracoronary delivery of mesenchymal stem cells and quanti- fication of resulting myocardial infarction by cardiac magnetic resonance. Circ Heart Fail 2010; 3: e5-6.

43. Tang XL, Rokosh G, Sanganalmath SK, et al. Effects of intracoronary infusion of escalating doses of cardiac stem cells in rats with acute myocardial infarction. Circ Heart Fail 2015 May 20 [Epub ahead of print].

44. Lalu MM, McIntyre L, Pugliese C, et al. Safety of cell therapy with mesenchymal stromal cells (SafeCell): a systematic review and meta-analysis of clinical trials. PLoS One 2012; 7: e47559.

45. Kooreman NG, Ransohoff JD, Wu JC. Tracking gene and cell fate for therapeutic gain. Nature Mat 2014; 13: 106-9. 